\title{
La terapia ocupacional y el proceso de aplicación de la estimulación sensorial-propioceptiva, en los recién nacidos prematuros de 0 a 3 meses
}

\author{
Karina Anastacia Gerula
}

\begin{abstract}
Resumen
Introducción: Este trabajo se realizó en el Hospital Materno Infantil "San Pablo", dentro del servicio de Neonatología, con un grupo de madres y sus hijos prematuros de 0 a 3 meses.
\end{abstract}

Objetivo: describir dentro de la disciplina de terapia ocupacional, el proceso de aplicación de la estimulación sensorial propioceptiva, determinando que beneficios aporta y que herramientas utilizar.

Material y Método: El estudio fue descriptivo- Cuasi experimental, y Transversal con un corte en el tiempo desde febrero a noviembre del 2018. En cuanto al material y método para su desarrollo se utilizó la encuesta y la entrevista a las madres, se elaboraron instrumentos, fichas de anamnesis, fichas de evaluación por áreas, fichas de seguimiento, teniendo como base la "Escala de Michigan", las Áreas evaluadas fueron: Motor Fino y Grueso, social afectivo y comunicación y lenguaje. Dividido en dos grupos con tratamientos diferentes, (uno recibió la estimulación sensorial-propioceptiva y las madres participaron de los talleres de forma activa desde la internación de sus hijos), el otro grupo solo tuvo una anamnesis original y sin estimulación sensorial propioceptiva, ni talleres).

Resultados: Dentro del resultado de las evaluaciones realizadas a los dos meses y tres meses, dividido en dos etapas, en una primera etapa de 0 a 2 meses se registraron, en el grupo activo: en el área motor grueso un $57 \%$ de respuestas positivas, en Motor fino $60 \%$, en comunicación y lenguaje $73 \%$ y en social afectivo $80 \%$, contra un $37 \%, 27 \%, 37 \%$ y $33 \%$ de respuestas positivas y pobres respectivamente en el grupo pasivo y en la segunda etapa de tres meses se registraron Motor Grueso 67\%, fino 70\%, Comunicación y lenguaje $80 \%$ y social afectiva $90 \%$, contra un $19 \%, 25 \%, 19 \%$ y $22 \%$ de respuestas positivas en el grupo pasivo.

Conclusiones: Se pudo visibilizar los beneficios que la estimulación sensorial temprana y propioceptiva aporta, en una mejor calidad de vida de los Recién

1. Universidad del Centro medico Batista, Paraguay.

E-mail: karinagerula36@gmail.com

DOI: $10.26885 /$ rcei.foro.2019.259

Trabajo publicado en acceso abierto bajo Licencia Creative Commons. 
Nacidos Prematuros; así como el papel fundamental que ejerce la madre o cuidador principal, en el aprendizaje y aplicación de estas técnicas. Resultando que los masajes y la estimulación sensorial propioceptiva son herramientas útiles y de muy bajo costo, que contribuyen en la recuperación de los recién nacidos prematuros que disminuye su estadía en la unidad de terapia intensiva neonatal, les ayuda a subir más rápido de peso, alimentarse del pecho materno en forma adecuada y mejorar positivamente en las diferentes áreas evaluadas.

Palabras clave: recién nacido prematuro, estimulación sensorial, masaje de tacto profundo.

\section{Referencias}

Federación Estatal de Asociación de Profesionales de Atención Temprana. (2005). El libro blanco de atención temprana. Madrid: ARTEGRAF.

Shelley, M. (2006). Terapia ocupacional en pediatría, proceso de evaluación. Madrid: Médica panamericana.

Polonio López, B. (2012). Terapia ocupacional en la infancia. Buenos Aires: Panamericana. 